\title{
Awareness of management of hyaluronic acid induced visual loss: A British National Survey
}

\author{
Varajini Joganathan ${ }^{1} \cdot$ Sabrina Shah-Desai ${ }^{2}$
}

Received: 1 June 2019 / Revised: 11 November 2019 / Accepted: 28 November 2019 / Published online: 17 February 2020

(c) The Author(s), under exclusive licence to The Royal College of Ophthalmologists 2020

\begin{abstract}
Background Visual loss from aesthetic hyaluronic acid filler injections is an under-reported complication. Our study surveyed British Oculoplastic consultants and Consultant members of the British Eye Emergency Care Society, on their awareness of visual vascular complications of dermal fillers and its emergency management.

Methods A prospective survey of the members of the British Oculoplastic Society and the British Eye Emergency Society, using the Survey Monkey platform.

Results There were 53 responses. Eighty five percent of responders were aware of ophthalmic artery occlusion and visual loss as a recognised complication of hyaluronic acid based fillers. Six respondents had encountered at least one case of visual loss associated with HA fillers. Approximately $27 \%$ of the respondents had some experience of the recommended appropriate management of this complication. Majority of these practitioners did not have local management guidelines for this complication (88\%) nor were they aware of guidance to manage the complication $(75 \%)$.

Conclusions This survey captures the current experience of British Ophthalmologists and Oculoplastic Surgeons in the management of visual vascular complication with dermal fillers. This may relate to the rarity of this complication. Although there is an awareness of visual loss as a complication from aesthetic dermal fillers, there appears to be a lack of knowledge of current management guidelines across Eye Specialists in UK. It is important for practitioners to be able to recognise and manage vascular compromise and further study recommendations are made.
\end{abstract}

\section{Introduction}

The use of dermal fillers has increased worldwide in the field of soft tissue augmentation and as a result reports of vascular complications have also increased [1]. Hyaluronic acid (HA) based dermal fillers are the most commonly used fillers in the field of aesthetics practice [2]. HA is a glycosaminoglycan and a chief component of the extracellular matrix and is mainly responsible for maintaining hydration in the dermis [3].

The percutaneous injection of HA fillers causes visual loss as a result of retrograde embolisation $[4,5]$ through arterial vascular system and can be classified into six

Sabrina Shah-Desai

manager@perfecteyesitd.com

1 Southampton University Hospital, Tremona Road, Southampton, UK

2 Perfect Eyes Ltd, 121, Harley Street, London W1G 6AX, UK subtypes depending on the artery involved [5, 6]. Furthermore, blindness can be associated with adnexal periocular complications [7].

Blindness resulting from vascular occlusion related to HA filler is an ophthalmological emergency, it being the retinal equivalent of a stroke. To date there is no National Ophthalmology Guideline or literature of Level 1 evidence to support any particular interventional therapy.

Recommended measures include immediate ophthalmologic consultation, ocular massage, Timolol eye drops, diuretics, haemodilution (with hydroxyethyl starch), corticosteroids, calcium channel blockers, anticoagulation, and needle decompression of the anterior chamber [8].

A consensus on practical guidance was published in April 2018, with a set of guidelines for the practitioner to use in an emergency situation along with guidelines for specialists to use in a secondary care setting [9].

Recommendations of this consensus publication included an immediate transfer to an eye hospital or accident and emergency department, along with advice to give other specialist and practitioners of Ophthalmology and 
emergency care on the use of retrobulbar hyalase as a specialist intervention.

We set out to evaluate the awareness and implementation of these recommendations for the management of patients with blindness as a complication of HA filler use, through survey of the members of the British Oculoplastic Surgery Society (BOPSS) and the British Eye Emergency Care Society (BEECS).

\section{Materials and Methods}

A comprehensive survey was created on Survey Monkey ${ }^{\circledR}$ platform and offered to the members of BOPSS and BEECS through their societies' websites through a link. The survey link was valid for single response collection per responder. The survey was open for 4 months from October 2018 to January 2019 (Table 1).

The surveys consisted of six questions and are tabulated in Fig. 1.

\section{Results}

Fifty three practitioners successfully completed the survey. Reassuringly a majority (85\%) of our respondents were aware of ophthalmic artery and its branches being at risk to occlusion and subsequent visual loss from cosmetic dermal filler injections.

Majority of the practitioners surveyed did not have local management guidelines for this complication $(88 \%)$, nor were they aware of any evidence based protocol to manage this complication $(75 \%, 39$ respondents). Fourteen responders $(25 \%)$ reported knowledge of availability of evidence based protocol to manage visual loss related to HA filler injection in the periocular area.

There was significant heterogeneity in response to the management of blindness as a result of periocular HA use. Fifty-two out of fifty-three respondents completed question 4, which asked, 'What techniques have you used or would consider using in the management of ophthalmic artery occlusion as a result of periocular Hyaluronic filler injection?' Of those who answered, fifteen respondents (29\%) answered they would carry out anterior chamber paracentesis and 14 (29\%) respondents answered that they would use retrobulbar hyaluronidase, only 2 respondents had personal experience in administering this in the periocular area. The remaining respondent answered they would carry out other emergency measures such as, ocular massage, intravenous Acetazolamide and breathing into a paper bag. (Fig. 1). A majority of the respondents $(46,87 \%)$ were not familiar with administering retrobulbar hyaluronidase for visual loss secondary to aesthetic HA use. One respondent stated to have used hyaluronidase in the periocular area for visual loss complication, but not in the retrobulbar location.

Six respondents reported to have encountered at least one case of visual loss associated with HA fillers and this seems

Table 1 Survey on the use of periocular Hyaluronic acid fillers.

1. Are you aware of ophthalmic artery occlusion being a potential complication of periocular Hyaluronic filler injection?

Yes

No

2. Do you or your department have a local management guideline for this complication?

Yes

No

3. Are you aware of any evidence based guidance to manage this complication?

Yes

No

4. What techniques have you used or would consider using in the management of ophthalmic artery occlusion as a result of Periocular Hyaluronic filler injection?

Breathing into bag

Ocular massage

Intravenous acetazolamide

Anterior chamber paracentesis

Retrobulbar hyaluronidase

Other

5. Are you familiar with the use of retrobulbar Hyaluronidase for this complication?

Yes

No

6. How many of these cases have you encountered in your practice over the last 5 years?
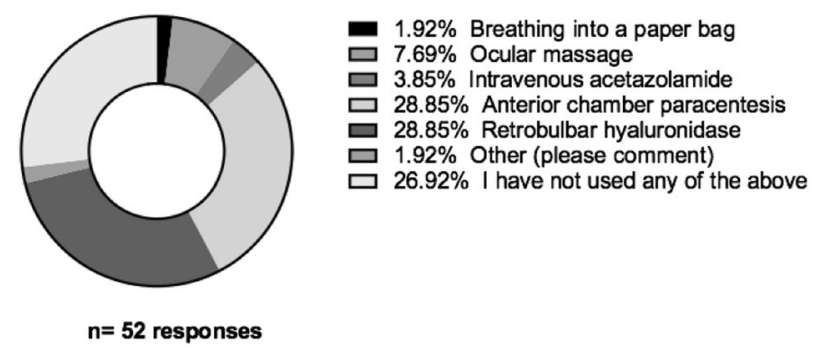

Fig. 1 Heterogenous responses (52 out of 53 practioners) to the management of acute visual loss as a result of vascular compromise following Hyaluronic acid filler injection. 
to contradict the rarity of this reported complication. In addition to this, one respondent reported 2 cases of visual loss fillers which they believed to have been from Sculptra (poly-lactic acid). This further highlights that the incidence of visual complication may be higher than that has been previously reported.

\section{Discussion}

Visual complication related to dermal fillers is defined as any impairment or loss of vision (temporary or permanent) secondary to central retinal or branch retinal artery occlusion occurring as a direct consequence of percutaneous injection for aesthetic treatment [8]. Visual loss remains a subset of vascular complications related to dermal fillers as it results in significant morbidity from an elective, aesthetic procedure. Visual loss was first reported as a complication relating to facial injection by Van Bahr [10] and since then 98 cases have been identified worldwide [11].

\section{Visual complications and outcomes}

Visual loss is often immediate and occurs within seconds [12] and depending on which artery is occluded, vision loss can be classified into six subtypes:[5, 6] ophthalmic artery occlusion, generalised posterior ciliary artery occlusion with relative central retinal artery sparing, central retinal artery occlusion, branch retinal artery occlusion, anterior ischaemic optic neuropathy, and posterior ischaemic optic neuropathy.

Visual loss may be associated with four subtypes of adnexal complications such as ophthalmoplegia and ptosis [7]. Previous case reports in the English literature suggest that ptosis and ophthalmoplegia symptoms, in contrast to visual loss respond better to immediate treatment [7].

Of the 53 survey responders, 3 were involved in the management of a total of 6 patients with altered vision post HA filler injection. To date there is no published data in the English literature to describe the incidence of blindness as a complication of the HA fillers in the United Kingdom (UK). This may simply be related to the rarity of reporting of such complication and perhaps related to a lack of a UK based, national reporting and support group for this iatrogenic complication. The exact incidence of this devastating complication remains to be determined in other large centres, outside the UK where most of the literature currently stems from [12]. Vascular complications from all dermal fillers, not specifically to periocular have been quoted to be 0.001 [13]-0.05\% [14].

\section{HA Fillers and Hyaluronidase}

Materials such as autologous fat, calcium hydroxyapatite and poly-lactic acid amongst others are all used as dermal fillers [12]. HA is a glycosaminoglycan and a chief component of the extracellular matrix and is mainly responsible for maintaining hydration in the dermis [3] and is increasingly used in aesthetic practice worldwide [2].

The nature of the smaller diameter 400 microns and the availability of an enzymatic dissolver, Hyaluronidase, are potential reasons for authors of case reports to predict a slightly better outcome with HA filler associated vascular complications that non HA filer associated complications [4, 15]. Irrespective of this it is imperative to provide appropriate and immediate management.

\section{Clinical practice and outcomes}

Many ophthalmic practitioners (73\%), according to our survey do not have the appropriate experience in its management. Subsequently the treatment of this devastating disorder is heterogeneous as described by the survey (Fig. 1).

Supportive measures described and used anecdotally, for non-filler related retinal thromboembolism has been intuitively employed by practitioners, ophthalmologists and oculoplastic surgeons in the management of HA filler related blindness.

Lazzeri et al. [8], in their literature review in 2012, looking at 32 cases of vascular complications leading to blindness state that treatment measure such as lowering intraocular pressure by incision with a blade, IV diuretics, carbogen (5\% carbon dioxide and $95 \%$ oxygen) rebreathing into a bag alongside corticosteroids were all unsuccessful.

It has been reported that retrobulbar injection of hyaluronidase should be administered within $60 \mathrm{~min}$ of accidental retinal artery embolism with HA fillers [16], to be effective [17].

Many studies have failed to show reversal of vascular obstruction or restoration of visual function [1]. Whilst Chestnut [18], has reported on its efficacy, and others have recommended the treatment of HA associated visual loss with retrobulbar hyaluronidase [17].

In the light of no best consensus being recommended, the recommended management against permanent blindness is to offer prompt retrobulbar hyaluronidase by a trained practitioner. None of our 53 survey responders have given retrobulbar hyaluronidase in practice, including those who have described involvement with patients with visual alteration following HA filler administration in the periocular area.

Forty responders $(80 \%)$ did not have access to a local management protocol and a majority of $75 \%$ (39) responders were not aware of any evidence based guidance. This highlights the imperative need to education; training and research with regards to clinical management of HA associated visual loss. At the time of writing this manuscript, the authors were not aware of any national guidance or 
treatment protocol, although authors have made recommendations on the appropriate management $[1,6,12]$.

\section{Future considerations}

Our survey suggests that majority of UK eye specialists, who have to manage patients with visual loss secondary to HA fillers, do not have the appropriate experience.

Overall there is an increase in the number of patients being treated with dermal filler injections by aesthetic practitioners in UK.

Prospective collection of data recording the incidence of visual loss associated with aesthetic treatments will be facilitated by a national adverse events reporting registry.

Current expert recommendations may be unnecessarily burdening the NHS with the onus of managing acute complications from aesthetic treatments. All aesthetic practitioners should have emergency treatment kits (as recommended by The Aesthetic Complications Expert Group of The Journal of Clinical and Aesthetic Dermatology and Fakih-Gomez et al. [1]) and be trained to recognise and treat this complication. The development of specialist private referral networks for the emergency management of this complication may be a way forward.

\section{Summary}

\section{What was known before}

- Visual loss from Hyaluronic acid dermal fillers require timely and expert acute management.

\section{What this study adds}

- Ophthalmic and Oculoplastic Specialists are referred these complications but often are unaware of the recommended guidelines to manage this emergency.

- This survey captures the current experience of British Ophthalmologists and Oculoplastic Surgeons in the management of visual vascular complication with dermal fillers.

- It is important for practitioners to be able to recognise and manage vascular compromise and further study recommendations are made.

\section{Compliance with ethical standards}

Conflict of interest The authors declare that they have no conflict of interest.
Publisher's note Springer Nature remains neutral with regard to jurisdictional claims in published maps and institutional affiliations.

\section{References}

1. Fakih-Gomez N, Orte-Aldea MC, Poonja K, Khanna D. Hyaluronic Acid Filler Emergency Kit. Am J Cosmet Surg. 2019;118:71-6.

2. King M, Convery C, Davies E. This month's guideline: The Use of Hyaluronidase in Aesthetic Practice. J Clin Aesthet Dermatol. 2018;11:E61-8.

3. Hirsch RJ, Brody HJ, Carruthers JDA. Hyaluronidase in the office: a necessity for every dermasurgeon that injects HA. J Cosmet Laser Ther. 2007;9:182-5.

4. Park SW, Woo SJ, Park KH, Huh JA, Jung C, Kwong OK. Iatrogenic retinal artery occlusion caused by cosmetic facial filler injections. Am J Ophthalmol. 2012;154:653-62.

5. Park KH, Kim YK, Woo SJ, Kang SJ, Lee WK, Korean Retina Society. Iatrogenic occlusion of the ophthalmic artery after cosmetic facial filler injections: a national survey by the Korean Retina Society. JAMA Ophthalmol. 2014;132:714-23.

6. Zhu GZ, Sun ZS, Liao WX, Cai B, Chen CL, Zheng HH, et al. Efficacy of retrobulbar hyaluronidase injection for vision loss resulting from hyaluronic acid filler embolization. Aesthetic Surg J. 2017;38:12-22.

7. Myung Y, Yim S, Jeong JH, Kim BK, Heo CY, Baek RM, et al. The classification and prognosis of periocular complications related to blindness following cosmetic filler injection. Plast Recon Surg. 2017;140:61-4.

8. Lazzeri D, Agonstini T, Figus M, Nardi M, Pantaloni M, Lazzeri S. Blindness following cosmetic injection of the face. Plast Reconstructive Surg. 2012;129:995-1012.

9. Humzah MD, Ataullah S, Chiang C, Malhotra R, Goldberg R. The treatment of hyaluronic acid aesthetic interventional induced visual loss (AIIVL): a consensus on practical guidance. J Cosmet Dermatol. 2019;18:71-6.

10. von Bahr G. Multiple embolisms in the fundus of an eye after an injection in the scalp. Acta Ophthalmol (Copenh). 1963;41:85-91.

11. Belezany K, Carruthers JDA, Humphrey S, Jones DJ. Avoiding and treating blindness from fillers: a review of the world literature. Dermatologic Surg. 2015;41:1097-117.

12. Loh KTD, Chua JJ. Prevention and management of vision loss relating to facial filler injections. Singap Med J. 2016;57:438-43.

13. Cohen JL, Biesman BS, Dayan SH, DeLorenzi C, Lambros VS, Nestor MS, et al. Treatment of hyaluronic acid filler-induced impending necrosis wth hyaluronidase: consensus recommendations. Aesthet Surg J. 2015;35:844-9.

14. Beleznay K, Humphrey S, Carruthers J, Alastair C. Vascular compromise from soft tissue augmentation. J Clin Aesthetic Dermatol. 2014;7:6.

15. Chen Y, Wang W, Li J, Yu Y, Li L, Lu N. Fundus artery occlusion caused by cosmetic facial injections. Chin Med J. 2014;127:1434-7.

16. Carruthers J, Fagien S, Dolman P. Retro or Peri Bulbar injection techniques reverse visual loss after filler injections. Dermatol Surg. 2015;41(suppl 1):S354-7.

17. Fagien S, Carruthers J. Commentary on restoration of visual loss with retrobulbar hyaluronidase injection after hyaluronic acid filler. Dermatol Surg. 2018;44:437-43.

18. Chesnut C. Restoration of visual loss with retrobulbar hyaluronidase injection after hyaluronic acid filler. Dermatol Surg. 2018;44:435-7. 